\title{
A CONTRIBUTION TO PREDICT THE STRUCTURAL DYNAMICS OF MOUNTED SHORT GLASS FIBER REINFORCED THERMOPLASTIC COMPONENTS
}

\author{
ALEXANDER KRIWET ${ }^{1}$, MARKUS STOMMEL ${ }^{2}$ \\ ${ }^{1}$ NVH Powertrain, Mercedes-Benz AG \\ Stuttgart, Germany \\ alexander.kriwet@daimler.com \\ ${ }^{2}$ Leibniz-Institute for Polymer Research \\ Dresden, Germany \\ stomme1@ipfdd.de
}

Key words: joints, structural dynamics, thermoplastics, stiffness, damping.

\begin{abstract}
At the state of art, current simulative methods modelling joint properties as fixed interaction between components or concentrate on the characterization of the joint stiffness and damping of coupled metallic structures. In the current work, the developed thin layer interaction (TLI) method was applied to study the influence of the joint stiffness and damping of plastic components coupled with metallic structures.

The joint stiffness and damping of different coupled metallic-plastic joint structures was characterized experimentally and was used to fill the reduced transverse shear stiffness and damping tensors of the numerical TLI model.

The TLI model was used to determine the structural dynamics behavior of a short glass fiber reinforced plastic engine bracket mounted to a steel block. The comparison of experimental and simulative results shows a better correlation under the usage of the TLI model compared to a fixed modeled joint behavior. As result, the TLI model allows a better prediction of the structural dynamics of coupled plastic components. Further researches focus on the accurate experimental estimation of the boundary conditions regarding excitation and mounting of the plastic components. Thus, a more realistic modelling of the numerical joint boundary conditions is possible for an improved simulation of the structural dynamics of plastic components.
\end{abstract}




\section{INTRODUCTION}

Short-fiber-reinforced-plastics (SFRP) improve the structure-borne sound radiation and sound transmission of force-transmitting components in modern powertrains under NoiseVibration-Harshness (NVH) aspects [1-3]. Thereby, engineering plastics show a strong increased damping behavior compared to metals and thus approach to the area of best material performance under NVH aspects [4,5]. A suitable example is an engine bracket made of polyamide 6.6 with $50 \mathrm{wg}$.- $\%$ glass fiber reinforcement (PA66-GF50). In modern vehicles, the engine bracket is mounted to the crankcase and transmits the structure-borne noise into the vehicle structure [6].

Current experimental investigations on plastic components show, that the mounting conditions influence the joint behavior and thus the structural dynamics. This leads to a frequency and amplitude shift with corresponding mode shape transformation.

However, established numerical methods for simulation of mounted components focus on static, fixed boundary conditions, due to reduced calculation capacity. In general, this approach is not sufficient to consider dynamic mounting conditions, like varying tightening torque or frequency dependent joint properties. As result, the present study focuses on an advanced approach to consider dynamic mounting conditions in the simulation.

First, the state of art regarding the numerical and experimental joint methods is presented. Thus, the constitutive equation to describe the joint behavior is reconsidered to regard joint

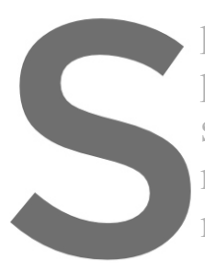
properties depending on the provide experimental joint simulations of the structural numerical joint method method. In this context, the
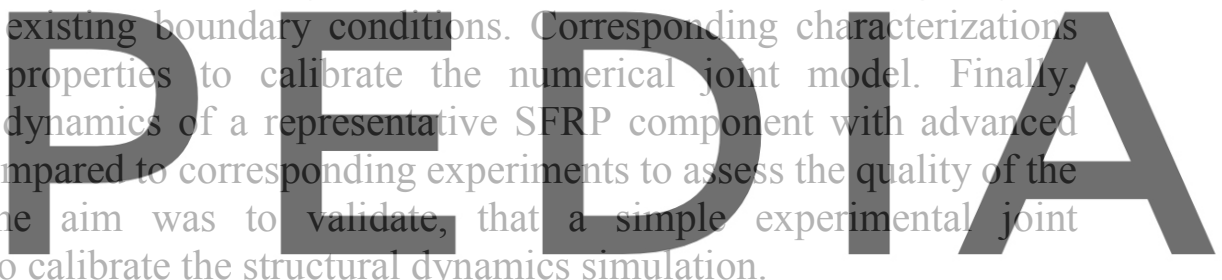

characterization is sufficient to calibrate the structural dynamics simulation.

Register for frea at htp\$twww.scipedia.com to download the version without the watermark

\subsection{Simulative methods}

The most common approach to consider an interaction between two components in a coupled FE based simulation is a fixed interaction. In this case, the displacement in the interaction zone of the components are set equal. The main disadvantage of this method is, that strong different material behavior of the coupled components can leads to strong different response behavior of the structure [7].

As results, advanced numerical methods consider a gradient of the displacement in the interaction between the coupled components. Thereby, the properties of the interaction zone are weighted with respect to the properties of the coupled components. In this context, a masterslave relation between the coupled components is defined. In this case, the displacement of the slave is governed by the motion of the master component. Further and comparable methods can be found in $[7,8]$.

The main disadvantage of all presented methods are, that they are limited to the defined interaction law or properties of the components. Advanced methods to consider dynamic joint 
properties focus on the approach of a material gradient in the interaction zone [9-11]. Thereby, the so-called Thin-Layer-Element (TLE) method is used. In general, TLEs are hexahedron elements, with a very small characteristic length/width-to-depth ratio $[12,13]$. They are modelled as continuum elements in the simulation, so that it is possible to associate these elements with a continuum equation to consider dynamic joint properties in the interaction zone.

In this context, two methods are established to calibrate the numerical joint properties: a purely numerical parameter optimization study [14] and a calibration of the simulation with experimental determined joint properties $[9,15]$.

\subsection{Experimental methods}

In general, there are a few number of established methods for an experimental determination of joint properties. Sanati et al. [16] and Eriten et al. [17] give an overview of current joint models with corresponding experimental characterization methods.

In this context, all existing methods have in common that they are based on the assumption of a multiple Degree-of-Freedom (DOF) spring-mass-damper model. For the evaluation of the structural dynamics response and thus the joint properties, the hysteresis loop approach is usually used. Thereby, the potential energy and the dissipated energy per cycle at resonance is evaluated [9,15-18].

However, existing research show, that the determined joint properties are significantly depending on the existing boundary conditions. Eriten et al. [17] showed, that the resulting experimental joint prop of the system and the rou

[16] showed, that the jo excitation. Comparabl investigations showed, that a reliable determination
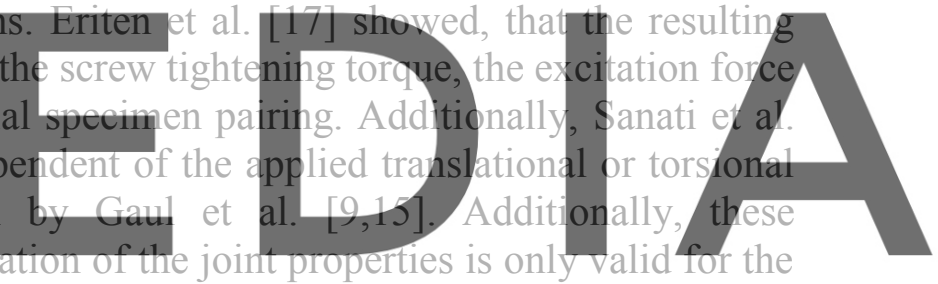
micro-slip area. Thereby, the response of the joint resonator is approximately linear, due to

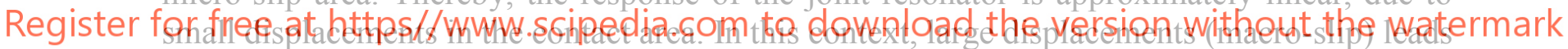

to an insufficient joint parameter extraction. As result, advanced researches like shown from Scheel et al. [18] concentrate on the determination of the joint behavior for non-linear effects.

However, existing researches to determine the effect of the joint properties focus in general on metallic specimen coupling. Joint properties of coupled plastic-metallic specimens are generally unknown.

\section{PROPOSED APPROACH}

\subsection{Advanced numerical joint behavior}

The TLE method allows to consider joint properties depending on the existing boundary conditions. Thereby, a TLE is placed in the simulation between the components to be joined and assigned with a constitutive equation. Existing investigations are based on a linear elastic, orthotropic material model assigned to the TLEs with the assumption, that the tangential parameters have a major influence on the joint behavior and the normal parameters have a little influence $[9,15]$. Following, the constitutive equation for the TLEs is defined as 


$$
\left[\begin{array}{l}
\sigma_{11} \\
\sigma_{22} \\
\sigma_{33} \\
\sigma_{44} \\
\sigma_{55} \\
\sigma_{66}
\end{array}\right]=\left[\begin{array}{cccccc}
C_{11} & C_{12} & C_{13} & 0 & 0 & 0 \\
& C_{22} & C_{23} & 0 & 0 & 0 \\
& & C_{33} & 0 & 0 & 0 \\
& & & C_{44} & 0 & 0 \\
& & & & C_{55} & 0 \\
& & & & & C_{66}
\end{array}\right]\left[\begin{array}{l}
\varepsilon_{11} \\
\varepsilon_{22} \\
\varepsilon_{33} \\
\varepsilon_{44} \\
\varepsilon_{55} \\
\varepsilon_{66}
\end{array}\right]
$$

with $C_{i j}$ as modulus, $\varepsilon_{i j}$ as strain vector and $\sigma_{i j}$ as resulting stress vector and $i, j=\{1 \ldots 6\}$. Under the assumption, that no joint parameters exist parallel to the contact interface and for inplane shearing, the components $C_{11}, C_{22}$ and $C_{44}$ vanish. Furthermore, the off-diagonal terms vanish under the assumption, that no transversal contraction exist in the interface $[9,15]$. Under these assumptions, it is possible to reduce equation (1) to

$$
\left[\begin{array}{l}
\sigma_{n} \\
\sigma_{S} \\
\sigma_{t}
\end{array}\right]=\left[\begin{array}{lll}
C_{n n} & 0 & 0 \\
& C_{S S} & 0 \\
& & C_{t t}
\end{array}\right]\left[\begin{array}{l}
\varepsilon_{n} \\
\varepsilon_{S} \\
\varepsilon_{t}
\end{array}\right]=C \varepsilon,
$$

with $C_{n n}$ as normal or penalty modulus and $C_{S S}=C_{t t}$ as tangential modulus. This can be described as Thin-Layer-Interaction (TLI) method. Thus, a TLI is placed in the simulation between the components to be joined. This is an established method to consider dynamic, userdefined joint properties in the simulation [19].
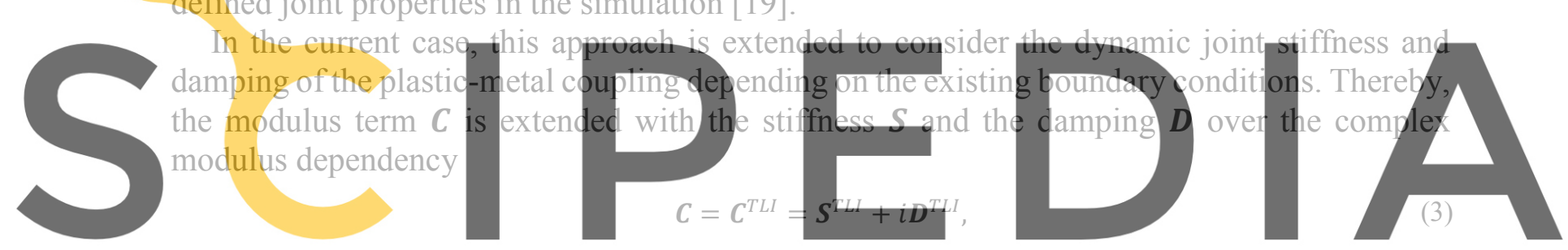

with $i$ as complex number. Thus, it is possible to implement user-defined equations to

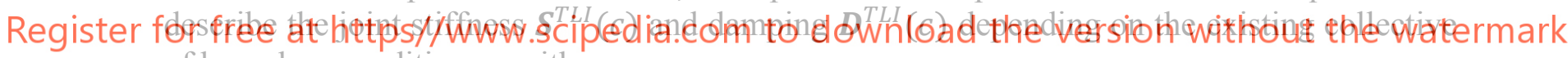
of boundary conditions $c$ with

$$
\boldsymbol{C}^{T L I}(c)=\boldsymbol{S}^{T L I}(c)+i \boldsymbol{D}^{T L I}(c) .
$$

As result, $\boldsymbol{S}_{j}^{T L I}(c)$ and $\boldsymbol{D}_{j}^{T L I}(c)$ represent experimental determined joint stiffness and damping values to calibrate the TLI.

\subsection{Experimental Joint Characterization}

To determine experimental values for the joint stiffness and damping of coupled plasticmetallic specimens, a joint-resonator system is used, shown in figure 1 . This is justified due to the simple setup and rapidity to determine joint properties. Furthermore, the joint properties are determined by modal analysis in the linear-elastic microslip area $[9,15,18]$. The resonator system contains of two masses, which are connected over a joint between them. In this, the contact pair specimens for the characterization of the joint parameters are defined. A shaker excites one side of the resonator system and thus the system gets into resonance at specific frequencies of the coupled system. 


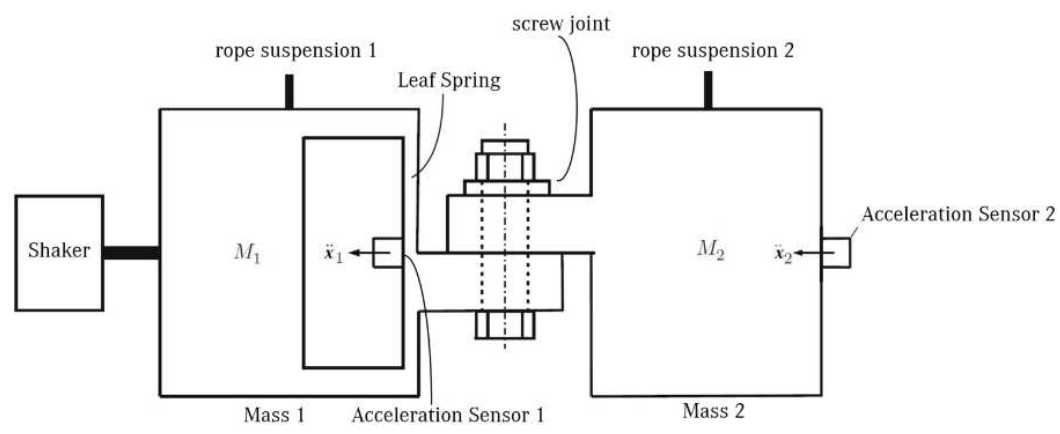

Figure 1: Schematically illustration of the joint-resonator system [9].

The procedure to determine the joint stiffness and damping is divided into three steps. First, the boundary conditions for the parameter extraction are defined.

In the second step, a frequency run-up excitation of the joint-resonator system with the shaker allows to evaluate the resonance frequencies of the coupled joint specimens.

Following, it is possible to determine the resulting displacement depending on the driven excitation force as hysteresis-loop. In this context, the slope of the hysteresis-loop equals the joint stiffness at resonance frequency and the enclosed surface equals the loss factor. The joint damping $E^{\prime \prime}$ can be evaluated as
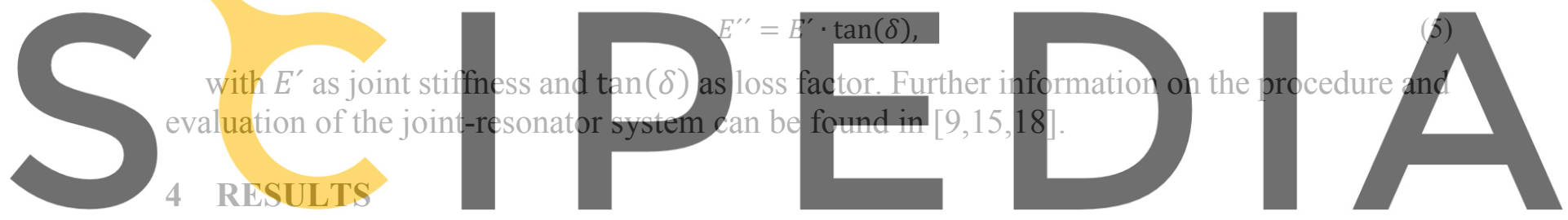

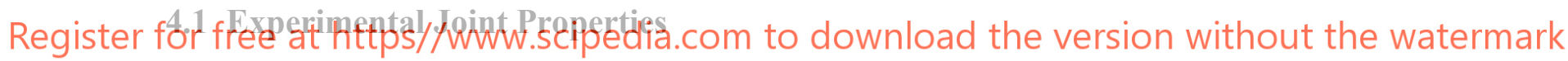

First, the boundary conditions for the parameter extraction were defined, see table 1.

Tabie 1: Overview of set local and giobal boundary conditions.

\begin{tabular}{ll}
\hline Boundary Condition & Parameter or Value \\
\hline Material Specimen 1 & PA66-GF50 \\
\hline Material Specimen 2 & Steel \\
\hline Screw Tightening Torque & $10,20,30 \mathrm{Nm}$ \\
\hline Excitation Frequency range & $1 \mathrm{~Hz}-2 \mathrm{kHz}$ \\
\hline Excitation Force & $1.2 \mathrm{~N}$ \\
\hline Temperature & $23{ }^{\circ} \mathrm{C}$ \\
\hline Humidity & $50 \%$ relative humidity $(\mathrm{rh})$ \\
\hline
\end{tabular}

To study the influence of dynamic mounting conditions, different screw tightening torques were applied with 10, 20 and $30 \mathrm{Nm}$, which represents realistic tightening torques of mounted SFRP components. Furthermore, the excitation frequency range to extract the joint properties 
was set from $1 \mathrm{~Hz}$ up to $2 \mathrm{kHz}$, thus $\mathrm{NVH}$ critical structural phenomena of the SFRP components are located in this range, especially for internal combustion engines [2].

In this context, the joint parameter extraction equals a parameter extraction under modal analysis. Thus, small excitation forces are sufficient to excite and capture the structural dynamics response of the system. Furthermore, higher excitation forces lead to insufficient nonlinearity structural dynamics $[9,18]$. As result, the excitation force was set to $1.2 \mathrm{~N}$. Moreover, the global boundary conditions focus on the temperature and humidity, thus the properties of the plastic specimens dependent on them. The global boundary conditions of temperature and humidity were set as normal climate with $23{ }^{\circ} \mathrm{C}$ and $50 \%$ rh in a climatic chamber, with regard to standard ISO 291 [20].

Next, a frequency run-up with resonance frequency analysis was performed. Corresponding, the hysteresis-loop evaluation determines the joint stiffness and loss factor. Following, figure 2 shows the average stiffness and loss factor of the characterized joint properties, depending on the applied tightening torque and the frequency.
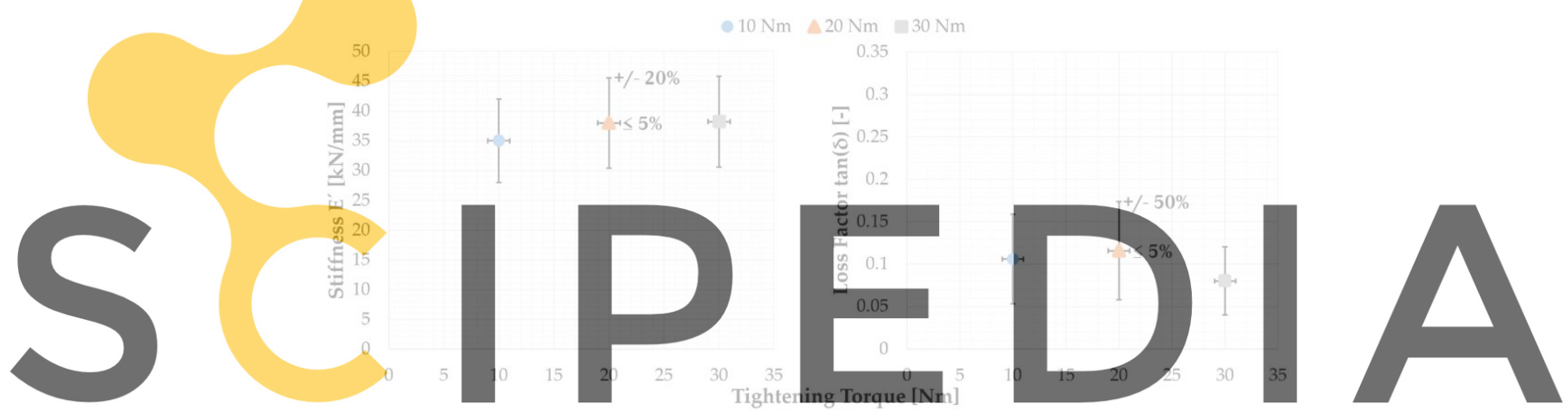

\section{Register for free at hittps//www.scipedia.com to download the version without the watermark}

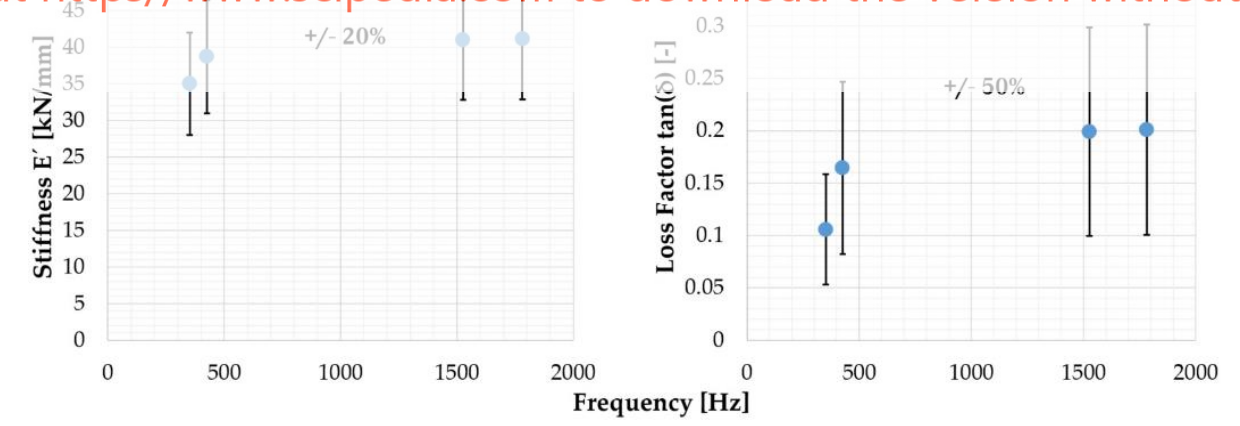

Figure 2: Average stiffness (left) and loss factor (right) at first resonance frequency range for 10, 20 and $30 \mathrm{Nm}$ tightening torque (top) and at tightening torque of $10 \mathrm{Nm}$ dependent of the frequency (bottom).

Figure 2 shows, that the joint stiffness tends to higher values with increasing tightening torque, whereas the loss factor in general decrease. However, with respect to all driven joint characterization procedures it can be shown, that the joint stiffness varies with an average deviation from about $+/-20 \%$ and the loss factor with $+/-50 \%$. This deviations can be traced 
back to the accuracy of the used method of the joint-resonator system, like shown in $[9,15]$. Moreover, the majority of all driven joint characterization procedures at $10 \mathrm{Nm}$ torque show a distorted hysteresis-loop. This leads to the assumption, that at $10 \mathrm{Nm}$ torque and $1.2 \mathrm{~N}$ excitation force, there is the start of the transition from micro- to macro-slip in the joint. Thus, only hysteresis-loops, which are not distorted were evaluated.

Furthermore, figure 2 shows the average stiffness and loss factor of the characterized joint properties at constant tightening torque of $10 \mathrm{Nm}$, depending on the frequency. In general, the joint stiffness increases with increasing frequency and leads to a saturation. This trend is comparable for the loss factor. Like shown before, the average deviations of the joint stiffness and loss factor are equal for the frequency-dependent properties thus the parameter extraction are based on the same database.

\subsection{Experimental Structural Dymamies Analysis}

In the current case, the engine bracket made of PA66-GF50 was used as suitable component to investigate the frequency response under varying mounting conditions with structural dynamics analysis (SDA). Figure 3 shows the used setup. Thereby, the engine bracket was mounted to a steel mass with significant higher weight compared to the plastic component. As result, the first resonance of the steel mass appears close to $2.6 \mathrm{kHz}$ thus the structural dynamics of the plastic component can be analyzed up to high frequency ranges without an overlay of the

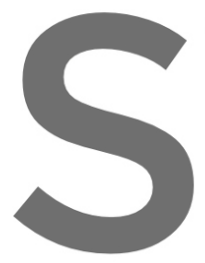
structural dynamics of

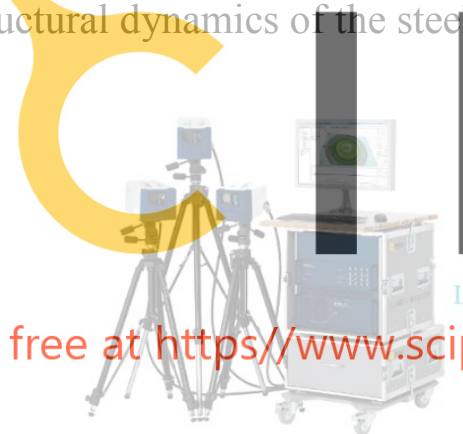

3D-Laservibrometer

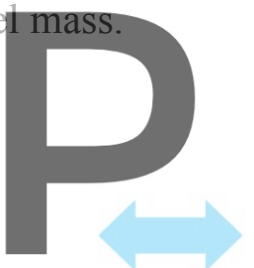

Register for free at https)/www.scipedia.com to download the version without the watermark
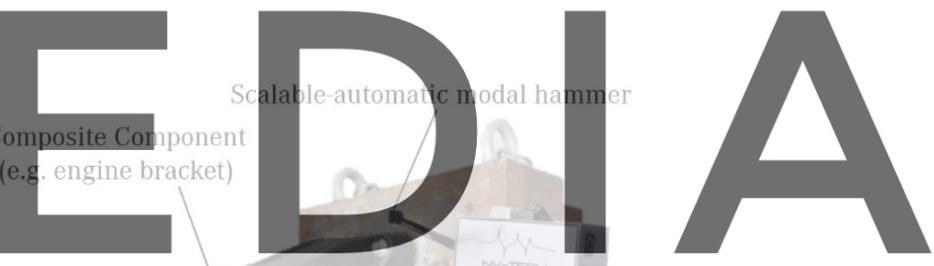

teei Miass

Figure 3: Setup for experimental component SDA.

In this context, the SDA of the engine bracket was performed for 10 and $30 \mathrm{Nm}$ screw tightening torque to set significant different mounting conditions in comparison. Moreover, the engine bracket was excited close to the area of the screw joints with a scalable automatic modal hammer and the frequency response was detected with a 3D-Laservibrometer during the SDA. Furthermore, the SDA was performed in a climatic chamber under normal climate from $23{ }^{\circ} \mathrm{C}$ and $50 \%$ rh with regard to standard ISO 291 [20].

Figure 4 shows the frequency response function (FRF) average for all laser-scanning points of the SDA of the plastic engine bracket for 10 and $30 \mathrm{Nm}$ screw tightening torque. Thereby, the amplitude was evaluated to the average acceleration response on the surface of the engine bracket in relation to the excitation force of the modal hammer. Figure 4 shows, that an increase of the tightening torque leads to a frequency shift in the direction of higher frequency ranges, 
whereas the amplitude decreases. Thus, it can be derived that an increase in the tightening torque causes an increase in the joint stiffness and reduce the joint damping. This shows a comparable correlation to the determined trend of the joint properties, shown in figure 2.

Furthermore, a comparison of the resonances was ensured by having a comparable mode shape at corresponding resonance frequency ranges. The mode shape analysis of the frequency range between 2 and $2.5 \mathrm{kHz}$ shows local structural dynamics close to the screw joints, shown in figure 4. As result, to study the influence of the joint behavior on the structural dynamics of the engine bracket in a simulation, only the frequency range $2-2.5 \mathrm{kHz}$ was considered.

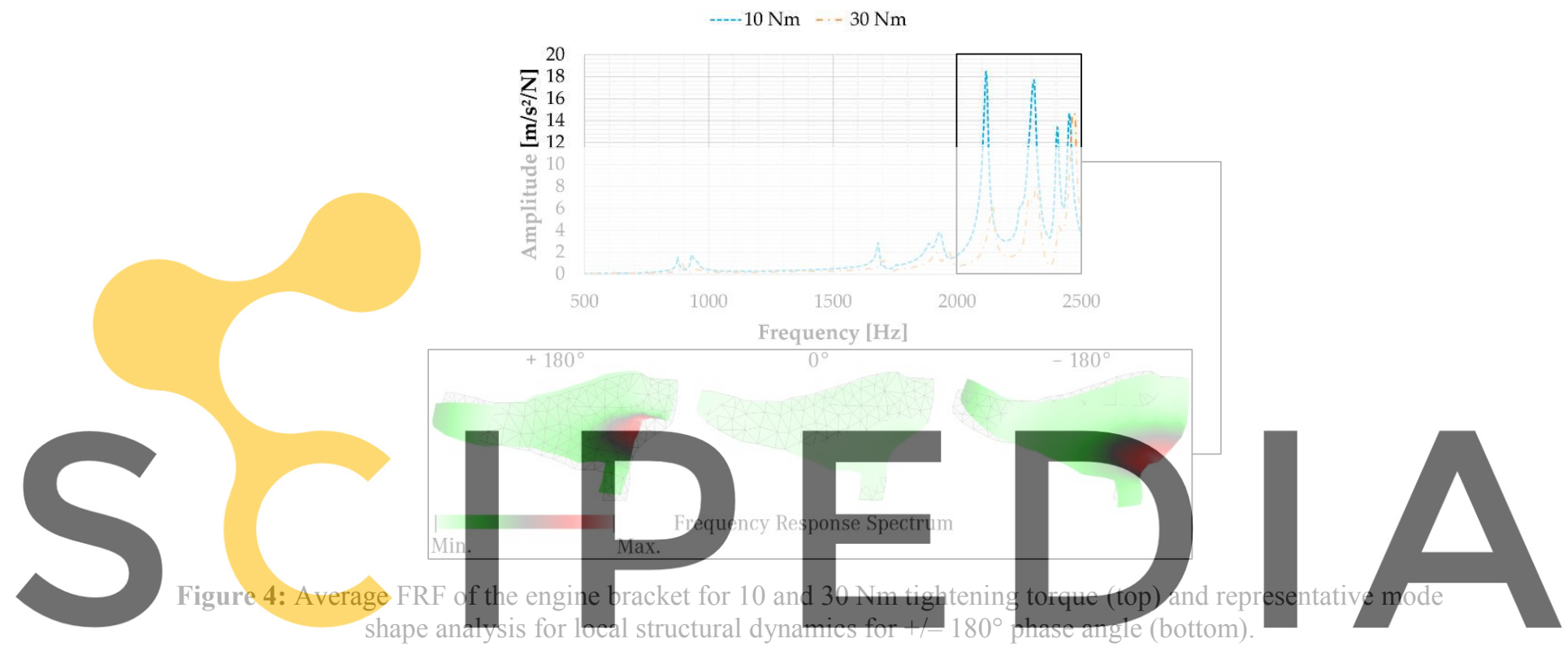

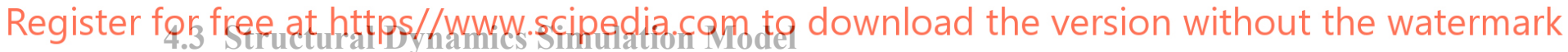

With respect to the experimental setup, the geometry of the plastic engine bracket and the steel mass was discretized into finite elements (FE) and the corresponding material behavior was assigned. The steel mass was discretized with 177,000 quadratic tetrahedron elements. The material model of all metallic components of the simulation model was defined as linear-elastic. In this context, the plastic engine bracket was discretized with 728,000 quadratic tetrahedron elements. This is justified by the applied Arbitrary-Reconsidered-Double-Inclusion (ARDI) material model of the SFRP engine bracket. In general, the ARDI model represents a two-step, multiscale homogenization approach and allows to consider temperature-, humidity-, frequency- and direction-dependent material properties. This ensures a sufficient material model for an accurate prediction of the structural dynamics of SFRP in a simulation [21].

Next, the numerical boundary conditions were set. The contact of all metal inserts to the plastic engine bracket was modelled as fixed constraint, due to the high strength of the press fit with negligible displacement [22]. Following, the joint behavior between the plastic component and the metal insert was modeled with the TLI method in the contact zone to the steel mass. Figure 5 shows the FE-discretized engine bracket and steel mass with applied TLI areas. 


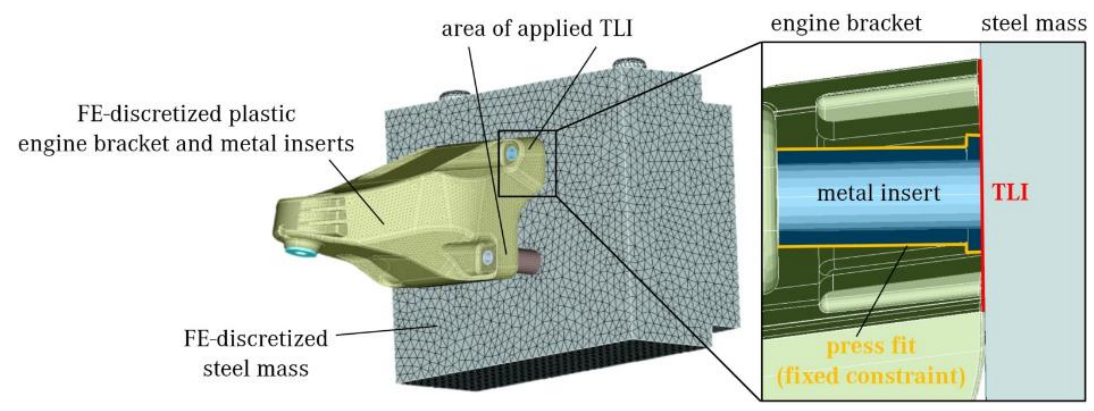

Figure 5: FE-discretized plastic engine bracket with metal inserts, steel mass and TLI areas.

To investigate the influence of the joint stiffness and damping on the structural dynamics of mounted SFRP in the simulation, the TLI method was integrated in the main equation of motion. Thereby, the main equation of motion is defined as

$$
F=M \ddot{x}+D \dot{x}+S x,
$$

with $\boldsymbol{F}$ as dynamic equilibrium force of the compound, $\boldsymbol{M}$ as mass matrix, $\boldsymbol{D}$ as damping matrix, $S$ as stiffness matrix, $x$ as resulting displacement vector, $\dot{x}$ as velocity vector and $\ddot{x}$ as acceleration vector. In the current case, this equation of motion is solved in the numerical simulation over a direct calculation with complex matrices to the resulting complex

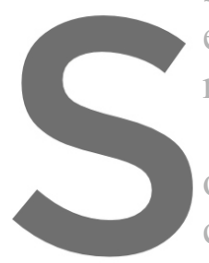
eigenvectors and eigen material properties depe

In general, the mass, of all included compon current case, the resulting stiffne
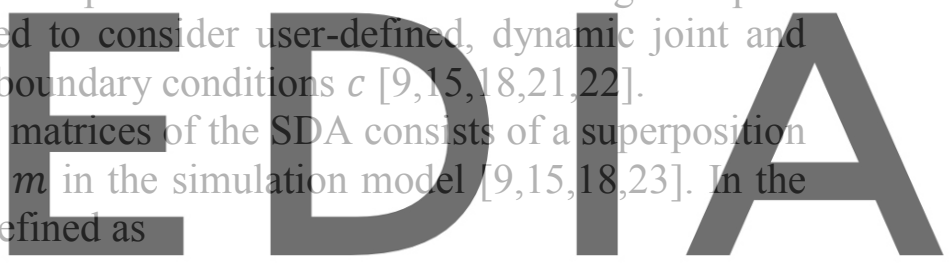

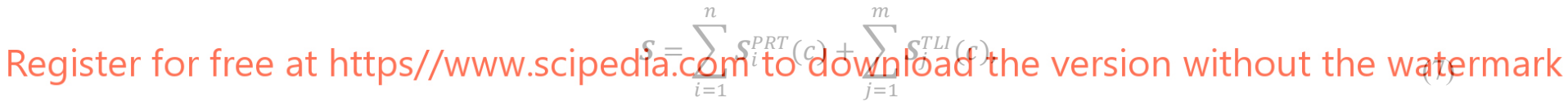

with $S_{i}^{P R T}(c)$ as stiffness of component $i$ and $S_{j}^{T L I}(c)$ as stiffness of the applied TLI $j$ depending on the existing boundary conditions $c$. Furthermore, this approach was adopted and used to describe the resulting damping tensor of the compound as well, with

$$
\boldsymbol{D}=\sum_{i=1}^{n} \boldsymbol{D}_{i}^{P R T}(c)+\sum_{j=1}^{m} \boldsymbol{D}_{j}^{T L I}(c) .
$$

In the current research, the main equation of motion was calculated for every frequency increment with the internal solver of the FE-Software ABAQUS. Furthermore, the userdefined, dynamic joint and material properties were updated for every frequency increment over an implemented FORTRAN user-subroutine. In this context, due to the high deviation of the determined experimental joint properties, a numerical joint parameter optimization study was performed, with regard to the experimental determined FRF of the plastic engine bracket. 


\subsection{Simulative Structural Dynamics Analysis}

To provide a better comparison of the TLI method with respect to the state of art, a simulation with fixed boundary conditions in the area of the joints was performed. Figure 6 shows the FRF of the experimental SDA of the engine bracket in comparison to the simulation with fixed boundary conditions at the joints.

In general, the comparison of the experimental and simulative FRF in figure 6 shows a good correlation. The resonance frequencies of the fixed simulation show an average deviation of $7 \%$ compared to the experiments. On the other hand, the amplitudes show an average deviation of $60 \%$. As result, the simulation with fixed boundary conditions at the joints combined with advanced material models is a sufficient method for a main prediction of the structural dynamics of the mounted SFRP component. However, with the usage of fixed boundary conditions, it exists no adjustable parameters to consider dynamic mounting conditions in the simulation, like different tightening torque.

Figure 6 shows the FRF of the experimental SDA of the engine bracket in comparison to the simulation with TLI method for $10 \mathrm{Nm}$ tightening torque. The comparison of the experimental and simulative FRF in figure 6 shows a good correlation. On the one hand, the resonances of the simulation show an average deviation of $8 \%$. Thus, the deviation is slightly increased compared to the fixed joint method. On the other hand, the amplitudes show an average deviation of $35 \%$.
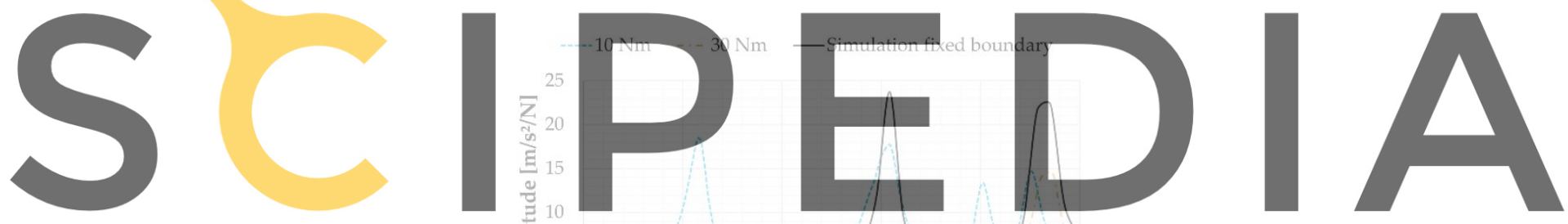

Register for free at https//www. scipedia.com to download the version without the watermark
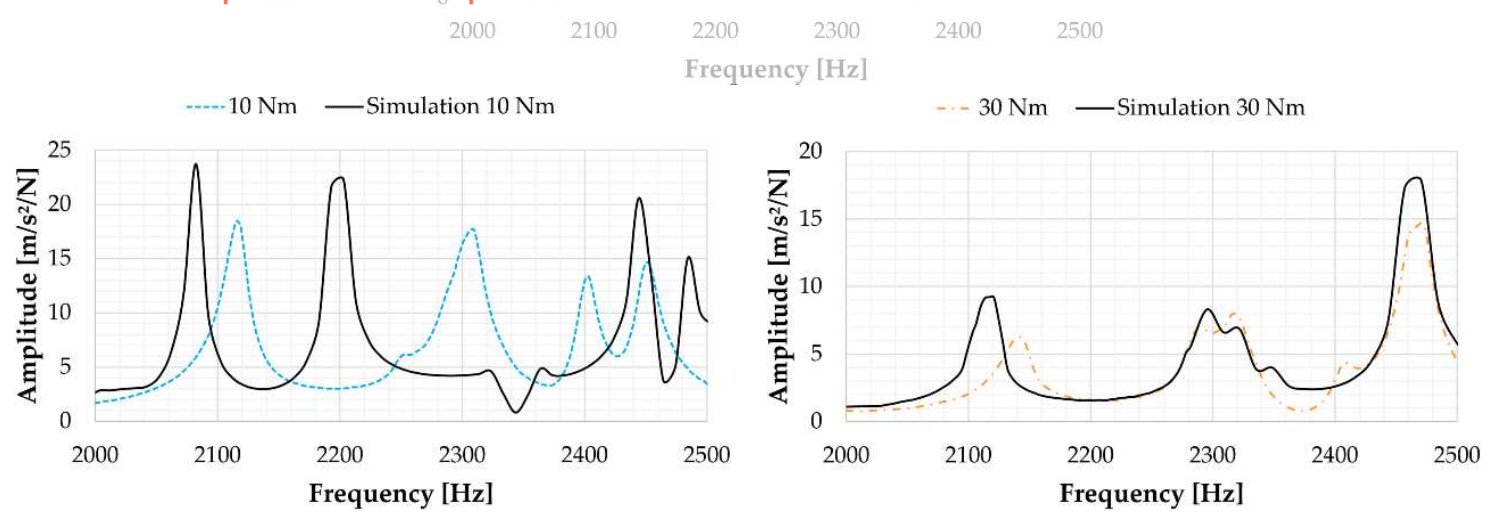

Figure 6: FRF of experimental SDA with 10 and $30 \mathrm{Nm}$ torque and simulation with fixed boundary conditions (top) and with 10 (left) and $30 \mathrm{Nm}$ (right) tightening torque and corresponding simulation with TLI (bottom).

Figure 6 shows the FRF of the experimental SDA in comparison to the simulation with TLI method for $30 \mathrm{Nm}$ tightening torque. The comparison of the experimental and simulative FRF 
for $30 \mathrm{Nm}$ torque shows a good correlation. Furthermore, the resonances show an average deviation of $2 \%$ and the amplitudes of $30 \%$. Thus the deviation is decreased compared to the TLI method with $10 \mathrm{Nm}$. As result, the TLI method allows a better prediction of the joint damping.

With respect to the experimental joint characterization it can be assumed, that micro- and macro-slip occur during the component analysis. Following, the simulation of the engine bracket with calibrated TLI consider the joint properties with deviations for a specimen behavior, see chapter 4.1. Thus, geometry-related influences like macro-slip, which are caused in the current case by the engine bracket, are not considered by the calibration of the TLI. As result, deviations in the resonances and the amplitudes occur in spite of the numerical joint parameter optimization study due to geometry-related effects. However, with respect to the used joint properties, the structural dynamics simulation of the plastic engine bracket with TLI method shows a good correlation to corresponding experiments. As result, the TLI method is suitable to consider dynamic mounting conditions in structural dynamics simulation of plastic components.

\section{CONCLUSIONS}

The advanced TLI method was presented to characterize the joint behavior of SFRP components over experimental determined joint stiffness and damping. In general, the comparison of the experimental and simulative FRF of the engine bracket shows a good correlation of the resonance frequencies and amplitudes. Deviations between the simulation and the experiment can be attributed to geometry-related effects during the component analysis. In this context, the TLI method provide dynamic joint stiffness and damping properties for varying mounting conditions of an engine bracket. Thus, dynamic mounting conditions were considered in the simulation and the influence on the structural dynamics of the plastic component were investigated.

With respect to the deviations between the experimental and simulative SDA, the TLI method allows an advanced contribution to predict the structural dynamics of mounted SFRP components. Further investigations focus on experimental structural dynamics characterization of the plastic components to determine and distinguish the geometry-related, overlaid structural dynamics influence. Thus, the experimental joint stiffness and damping can be recalibrated.

\section{REFERENCES}

[1] Stommel, M.; Stojek, M.; Korte, W. FEM zur Berechnung von Kunststoff- und Elastomerbauteilen, 2nd ed., Carl Hanser Verlag: München, Germany, (2018).

[2] Raschke, K.; Korte, W. Faserverstärkte Motorbauteile besser berechnen. Kunststoffe (2019) 109:184-189.

[3] Laak, M. op de; Hauth, M. Noch schneller zur Zylinderkopfhaube. Kunststoffe (2004) 94:126-130.

[4] Lakes, R. Viscoelastic Materials, Cambridge Univ. Press, (2009).

[5] Dong, L.; Lakes, R.S. Advanced damper with high stiffness and high hysteresis damping based on negative structural stiffness, Int. J. Solids Struct. (2013) 50:2413-2423. 
[6] Raschke, K.; Korte, W. Charakterisierung und FEM-Modellierung von kurzfaserverstärkten Kunststoffen zur Verbesserung der Vorhersage akustischer Eigenschaften von Strukturbauteilen, Plastics in Automotive Engineering (PIAE), (2018).

[7] Dassault Systèmes. Abaqus 6.11 Analysis User's Manual Volume V: Prescribed Conditions, Contraints \& Interactions (2011).

[8] Johnson, K. L. Contact mechanics, $9^{\text {th }}$ ed. Cambridge: Cambridge Univ. Press, (2003).

[9] Ehrlich, C.; Schmidt, A.; Gaul, L. Microslip Joint Damping Prediction Using Thin-Layer Elements, Dynamics of coupled structures, Proceedings of the $32^{\text {nd }}$ IMAC (2014) 1:239244.

[10] Dassault Systèmes. Abaqus Documentation 6.14 User Subroutine Reference Guide: UINTER (2014).

[11] Dassault Systèmes. Abaqus Verification Guide: 4.1.20 UINTER (2014).

[12] Desai, G. N.; Zaman, M. M.; Lightner, J. G.; Siriwardane, H. J. Thin-Layer Element for Interfaces and joints, Int. J. Num. and Analyt. Meth. In Geomechanics (1984) 8:19-43.

[13] Pande, G. N.; Sharma, K. G. On Joint/Interface Elements and Associated Problems of Illconditioning, Int. J. Numer. Anal. Methods Geomech. (1979) 3:293-300.

[14] Jalali, H.; Hedayati, A.; Ahmadian, H. Modelling mechanical interfaces experiencing micro-slip/slap, Inverse Problems in Science and Engineering (2011) 19:751-764.

[15] Gaul, L.; Schmidt, A.; Bograd, S. Experimentelle Ermittlung zur Werkstoff- und Fügestellendämpfung sowie deren Berücksichtigung in Finite-Elemente-Berechnungen, Nr. 877, Forschungsvereinigung Verbrennungskraftmaschinen e.V., Germany, (2008).

[16] Sanati, M.; Terashima, Y.; Shamoto, E.; Park, S. S. Development of a new method for joint damping identification in a bolted lap joint, J. Mech. Sci. Technol. (2018) 32:19751983.

[17] Eriten, M.; Lee, C. H.; Polycarpou, A. A. Measurements of tangential stiffness and damping of mechanical joints: Direct versus indirect contact resonance methods, Tribology International (2012) 50:35-44.

[18] Scheel, M.; Peter, S.; Leine, R. I.; Krack, M. A phase resonance approach for modal testing of structures with nonlinear dissipation, J. Sound and Vibration (2018) 435:56-73.

[19] Boer, H. de; Weustink, A. P. D.; Beukers, A.; Koussios, S. Modelling Interfaces and Bonded Joints, $16^{\text {th }}$ Int. Conf. Compos. Materials (2007).

[20] ISO 291:2008. Plastics - Standard atmospheres for conditioning and testing, International Organization for Standardization, Germany, (2008).

[21] Kriwet, A.; Stommel, M. Arbitrary-Reconsidered-Double-Inclusion (ARDI) Model to Describe the Anisotropic, Viscoelastic Stiffness and Damping of Short Fiber-Reinforced Thermoplastics, J. Compos. Sci. (2020).

[22] Yared, W. Characterization of the Influence Collective on the Simulative Modal Analysis of Plastic Components and Engine-Sub-Structures in the Engine-Transmission Unit, Master Thesis, Technical University Dortmund, Germany, (2019).

[23] Ehrlich, C.; Schmidt, A.; Gaul, L. Reduced thin-layer elements for modeling the nonlinear transfer behavior of bolted joints of automotive engine structures, Arch. Appl. Mech. (2016) 86:59-64. 